Research Article

\title{
Experiments on the Porch Swing Bearing of Michelson Interferometer for Low Resolution FTIR
}

\author{
Tuomas Välikylä and Jyrki Kauppinen \\ Department of Physics and Astronomy, University of Turku, 20014 Turku, Finland \\ Correspondence should be addressed to Tuomas Välikylä; tuomas.valikyla@utu.fi
}

Received 22 March 2013; Accepted 8 May 2013

Academic Editor: Joseph Rosen

Copyright (C) 2013 T. Välikylä and J. Kauppinen. This is an open access article distributed under the Creative Commons Attribution License, which permits unrestricted use, distribution, and reproduction in any medium, provided the original work is properly cited.

Porch swing bearing for the linear motion of the mirror in Michelson interferometer for mid-infrared low resolution Fourier transform spectrometer was studied experimentally using the modulation depth of the collimated laser beam. The mirror tilting was measured to be lower than $5 \mu \mathrm{rad}$ over $3 \mathrm{~mm}$ mirror travel using two different bearings assemblies. Additionally, the manufacturing tolerances of the bearing type were proved to be loose enough not to limit the interferometer application. These demonstrate that the porch swing without any adjustment mechanisms provides the sufficient motion linearity.

\section{Introduction}

Inexpensive and robust portable Fourier transform infrared (FTIR) spectrometers for gas analysers are still needed, because the existing solutions have some weaknesses like expenses, bulky size, or sensitivity to the temperature variations. The most sensitive component in FTIR spectrometer is the interferometer. We have aimed to design such an interferometer with $25 \mathrm{~mm}$ beam diameter and with $2 \mathrm{~cm}^{-1}$ resolution in the mid-infrared region. Numerous interferometer designs can be found, for example, from the papers by Jackson [1] and Kauppinen et al. [2] and from the book by Griffiths and de Haseth [3]. We have selected Michelson interferometer with plane mirrors, because it has only a few, even inexpensive, optical components. Using Michelson interferometer, it is possible to minimize the size and cost of the interferometer structure while keeping the beam diameter and the throughput constant. Additionally, we will not use any dynamic alignment of the optics which are commonly applied in most solutions.

One of the key parameters in interferometer design is the modulation depth of the interferogram. In Michelson interferometer built using plane mirrors, the modulation depth is practically mainly affected by the tilting of optics which causes an angle between the output beams from inter- ferometer. The shearing is the second major phenomenon determining the modulation depth. It is caused by the lateral shift of the output beams. With the plane mirror setup, the shearing is usually negligible because the plane mirrors do not shift the beams with each other. If the cube corners would be used as the end mirrors, the output beams would not tilt but they may be shifted with each other which would decrease the modulation depth especially when the size of the radiation source is finite. Maximizing the modulation depth sets the requirements for the movement of the mirror. The movable mirror has to remain parallel to the image of the second mirror formed by the beam splitter with extreme accuracy over the desired mirror travel. This linear motion of the mirror is achieved by a suitable bearing system which is reviewed quite throughly by Jackson [1]. The air bearings, both compressed air and magnetic suspensions $[4,5]$, provide very smooth movement and low friction but are usually bulky and expensive. Various sliding bearings, like syringes or rails and guide bar systems, have many advantages including low costs but, in general, they are quite sensitive to produce misalignments and can require special materials or extreme cleanliness in manufacturing [1].

The porch swing bearings, presented in Figure 1, have none of these disadvantages. They have quite a simple structure which can be made rather small. The porch swing 


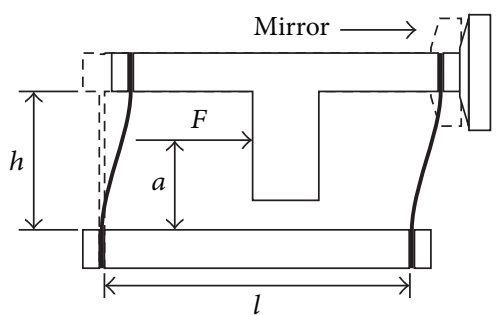

(a)

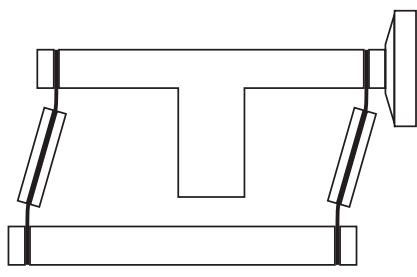

(b)

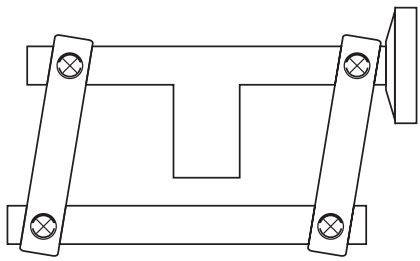

(c)

Figure 1: Side view of typical porch swing bearing assemblies for realizing the linear movement of the mirror. The drawing (a) shows flat springs as the flexure elements and the drawing (b) stiffener clamps in the springs. In the drawing (c), the flexure pivots are used. The driving force $\mathbf{F}$ is exerted to the upper arm.

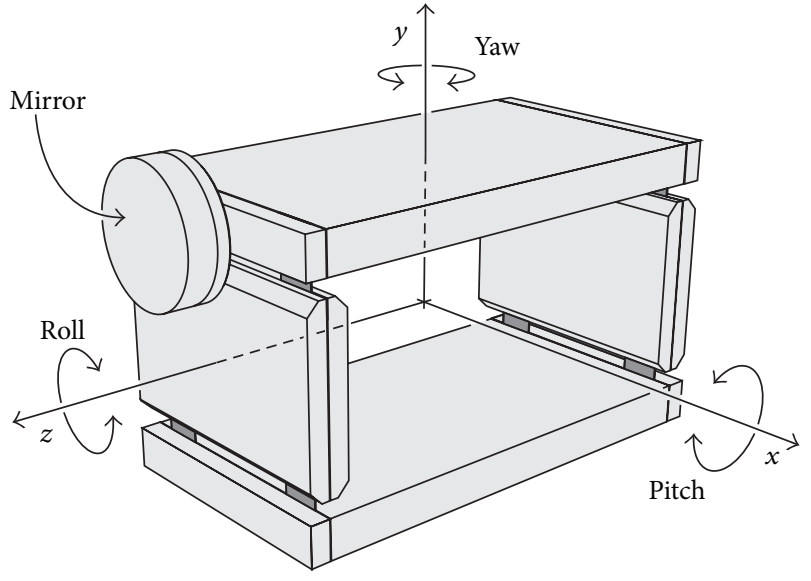

FIGURE 2: Type of the porch swing bearing used in the measurements and the definition of the directions of the coordinate axes and the labels of the rotations.

is a four bar parallelogram linkage where the pivots are usually flexure pivots, flat springs, or other flexure elements. They provide virtually frictionless driving and do not need lubrication. Additionally, they have no wear if they are loaded correctly.

According to Griffiths and de Haseth [3], the porch swing bearing was first used in an interferometer by Walker and Rex [6] in the 1970s. However, Jones [7] has mentioned that the porch swing mechanism for the linear motion is "well known," already in 1951. Walker and Rex used the flexure pivots as in Figure 1(c) in their bearing design. This kind of interferometer has been operated successfully in harsh environments like helicopters by Small et al. [8], in an air balloon by Huppi et al. [9], or during a rocket flight by Kemp and Huppi [10]. According to Hanel et al. [11], a kind of parallel spring suspended moving shaft was used in the interferometer in Nimbus III space flight in 1969. Wishnow et al. [12] have described an interferometer with porch swing driving for visible band imaging spectrometer used in the telescope. Many patents, as [13-17], are also related to the porch swing design. The porch swing driving is also used in a few commercial products, but they typically use a dynamic alignment system.
Because the linearity of the motion is essential, we have studied experimentally the tilting of our interferometer design which has a porch swing as in Figure 2. We will demonstrate nearly a perfect porch swing, where the modulation depth has almost no decrease over the $3 \mathrm{~mm}$ driving path. Additionally, we have quite good measurement results with the porch swing where all the parts of the bearing have normally achievable machining tolerances. The tilting was estimated using a Helium-Neon laser beam and its modulation depth.

In addition to the tilting, there are many other phenomena, like thermal expansion and stability in temperature changes, the force constant and the resonance frequencies of the mechanism, or the aging of the mechanics, which have to be considered in design. The lifetime of the flexure elements, typically made of steel, is normally very long if they are loaded correctly. However, these are out of the scope of this article, and we concentrate on the driving stability.

\section{Experiments on Motion of the Porch Swing Bearing}

2.1. Requirements for the Linearity. The maximum allowed decrease of the modulation depth sets the limits for the tilt angle which has to be achieved during a single scan and from scan to scan; hence, the signal strength is proportional to the modulation depth. The modulation depth of the circular output beam of the interferometer can be expressed as

$$
m=\frac{2 J_{1}(2 \pi D \alpha \nu)}{2 \pi D \alpha \nu} \approx 1-\frac{(2 \pi D \alpha \nu)^{2}}{8},
$$

where $D$ is the beam diameter, $\alpha$ is the tilt angle, $\nu$ is wavenumber or the reciprocal of the wave length, or $\nu=1 / \lambda$, and $J_{1}$ is the Bessel function of the first kind, and the uniform intensity distribution over the cross-section of the collimated infrared beam is assumed $[2,18]$. So, the modulation depth is roughly proportional to the square of the tilt angle which makes the interferometer very sensitive to the changes in the tilt angle. To keep the modulation depth above 0.95, the tilt angle should be less than about $14 \mu \mathrm{rad}$ with the beam diameter of $25 \mathrm{~mm}$ and the wavenumber of $3000 \mathrm{~cm}^{-1}$. This wave number is one common convention to report the modulation depth, and so we use it here also. 


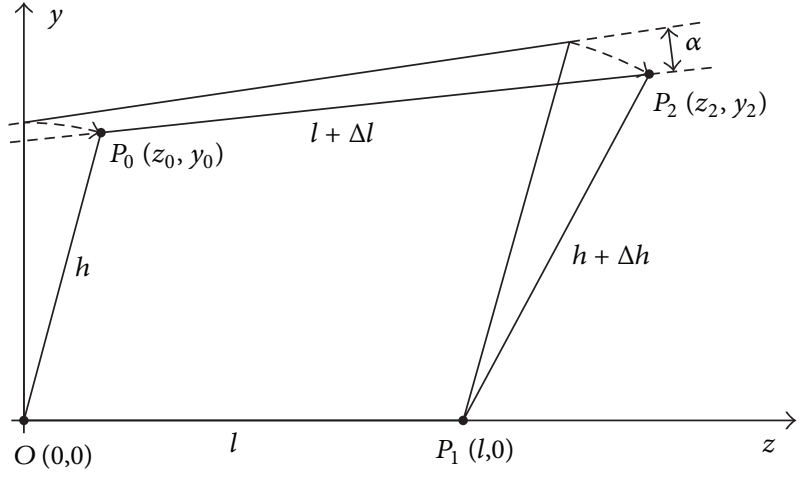

FIGURE 3: Side view of the porch swing structure with manufacturing errors $\Delta l$ in the arm and $\Delta h$ in the flexure lengths which produce the pitch angle $\alpha$ when the upper arm is pushed to $P_{0}$. The errors are exaggerated.

\subsection{Stiffening Clamps in the Flexures Reduce Parasitic Motions.} If the porch swing bearing is perfect, the arms stay parallel with each other during driving. The movable arm follows actually a circular trajectory but if the travel length is short, the motion is nearly linear. The imperfections of the porch swing structure cause the unwanted rotations of the movable arm and so tilting of the mirror during the travel. These undesired rotations are also called parasitic motions. When only the plane mirrors are used in the interferometer, the modulation depth is practically affected only by tilting around $x$ and $y$ axes or pitch and yaw rotations, respectively. The rotation around the $z$ axis and shifting on the $x y$-plane have either no or negligible effect on the modulation depth. Additionally, the arm shifts always in the $y$ direction because of the circular trajectory, but with small travel, this shift is negligible.

The length differences of the arms $\Delta l$ or the flexure elements $\Delta h$ cause the pitch rotation $\alpha$ of the arm as illustrated in Figure 3. The angle of the nonparallelism of the neutral axis of the flexures $\theta$ or the principal axis of the inertia of the flexures $\phi$ produce the yaw rotation $\beta$. The tilting is also resulted by any deviation of the driving force $\mathbf{F}$ from the ideal position which lies along a line which is parallel to the $z$ axis and runs through the center of the cross-section on the $x y$-plane of the porch swing. These errors are discussed in more detail in the appendix and in the literature [19-24]. In addition to the above errors, many extra possible sources of the parasitic motions are mentioned in the literature. These include, among others, the friction at the contact point of the driving force, the asymmetric mass distribution of the arm, gravity orientation, and variations of the spring material. As a summary, the porch swing is most sensitive to the unequal lengths of the arms $\Delta l$ and the angle between the principal axes of inertia $\phi$.

When the flat springs are used as the flexure elements, the parasitic rotations can been substantially decreased by the stiffening clamps in the middle of the springs as in Figures $1(\mathrm{~b})$ and $2[7,16,20,25]$. The clamps seem to make flexure elements more similar to each other and increase the stiffness in the $x$ direction which decrease the sensitivity to the tilting.
We have demonstrated the effect of the stiffening clamps by measuring the tilt angle as a function of the mirror displacement using the porch swing bearing which was constructed of two $l=50.0 \mathrm{~mm}$ long arms and four steel springs with $h=50.0 \mathrm{~mm}$ long flexible part. The springs were $12.7 \mathrm{~mm}$ wide and $0.30 \mathrm{~mm}$ thick. They were mounted at the end of the arms as pairs with distance of $2.7 \mathrm{~mm}$ between them. The configuration of the spring elements was as in Figure 2. The stiffening clamps were two pairs of aluminum plates with length of $25 \mathrm{~mm}$ which was the half of the spring length $h$. They were mounted in the halfway of the arms as in Figure 1(b). The lower arm of the porch swing was rigidly mounted on the table, and the upper arm could be pushed using a rod with fine threads. The light source was a red Helium-Neon laser whose beam was collimated to the plane wave. The coherence length of the laser light was roughly about $20 \mathrm{~cm}$, so it was not necessary to scan the interferometer exactly around the zero of the optical path difference. Instead, the zero of the mirror displacement $z_{0}$ was the equilibrium position of the spring elements. The optical setup is depicted in Figure 4. The end mirror M1 of the Michelson type interferometer was mounted on the top of the movable arm and the other optics on the table. The interference fringes were magnified by a diverging lens to a screen with a millimeter scale attached to it. We measured the distances $d$ between adjacent fringes on the screen as illustrated in Figure 5. The magnification of the lens was considered by scaling the distance values. The experiment was repeated with and without the stiffening clamps. The tilt angle can be calculated from the fringe distance $d$ as

$$
\alpha=\frac{k \lambda}{2 D}=\frac{\lambda}{2 d},
$$

where $k$ is the number of the fringes across the beam with diameter of $D$ and $\lambda$ is the wave length of the beam. If the fringe distances are measured orthogonally, the actual distance is

$$
d=\frac{d_{x} d_{y}}{\sqrt{d_{x}^{2}+d_{y}^{2}}} .
$$

Results of the tilt angles are presented in Figure 6. Without the stiffening clamps the tilt angle was about $90 \mu \mathrm{rad}$ after $4 \mathrm{~mm}$ translation, but with clamps the tilt always stayed well below $20 \mu \mathrm{rad}$. Thus, the results support the proposition that the clamps increase the stability of the moving arm.

2.3. Manufacturing Tolerances. We estimated the maximum allowed manufacturing tolerances of the porch swing bearings which had the typical dimensions $l / h=50 / 50, l / h=$ $100 / 50, l / h=150 / 50$, and $l / h=50 / 100$. The width of the bearings was $w=50 \mathrm{~mm}$. As the limit for errors, we used the conditions given in Section 2.1 or $m \geq 0.95$ and $\alpha \leq 14 \mu \mathrm{rad}$ which ensures that the bearing is suitable for FTIR interferometer.

The porch swing structure is most sensitive to the difference in the arm lengths $\Delta l$ and the angle $\phi$ between the ends of the arms. The greatest allowed arm length differences were 


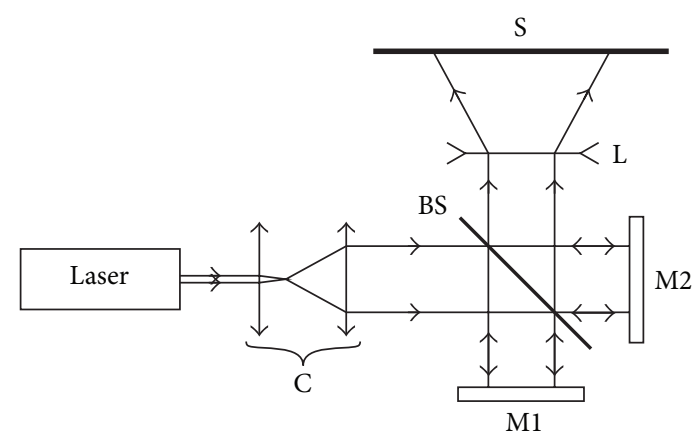

FIGURE 4: Measurement setup for studying the fringe pattern of the output beam of the interferometer. The beam from Helium-Neon laser was collimated in $\mathrm{C}$ and then pointed through the Michelson interferometer built from plane mirrors $\mathrm{M} 1$ and $\mathrm{M} 2$ and a cube beam splitter BS. The mirror M2 was rigidly mounted, and the mirror M1 was moved using the porch swing bearing. The diverging lens $\mathrm{L}$ was used to enlarge the fringe pattern on the screen $\mathrm{S}$ which had a millimeter scale attached to it.

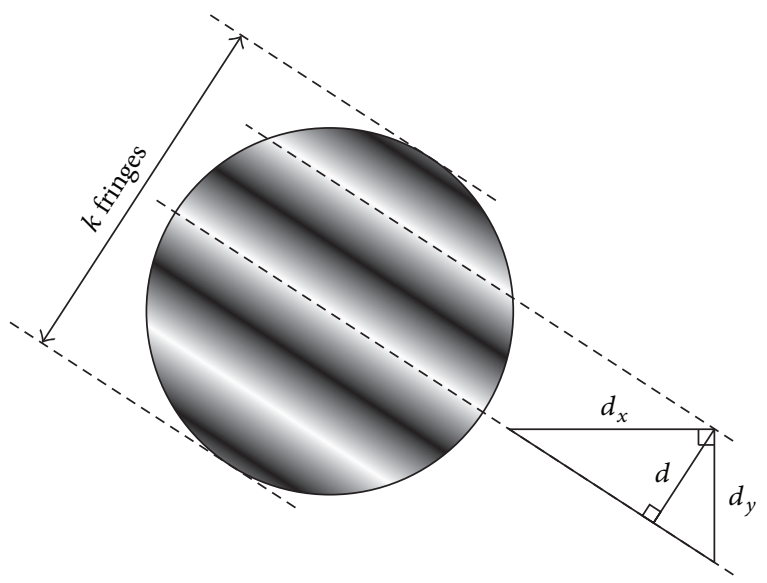

FIGURE 5: The tilt angle can be determined from the fringe pattern by measuring the distance $d$ between the adjacent fringes or counting the number of fringes $k$ across the beam. Both of these can also be measured orthogonally to obtain pitch and yaw rotations.

calculated using (A.la) and (A.lb). These values varied from $0.02 \mathrm{~mm}$ to $0.06 \mathrm{~mm}$ between the cases. The tolerance is loosening when the arm is extending. The nonparallelism of the arm ends causes angle $\phi$ between the principal axes of inertia of the flexures. The maximum allowed angles were from $230 \mu \mathrm{rad}$ to $680 \mu \mathrm{rad}$ according to (A.6). This corresponds to $0.01 \mathrm{~mm} \ldots 0.03 \mathrm{~mm}$ difference in the lengths of the opposite sides of the arm ends. However, by careful machining, the error $\Delta l \leq 0.01 \mathrm{~mm}$ at least is achievable. So, these tolerances do not seem to limit the usefulness of the bearing.

The largest allowed length difference of the flexures $\Delta h$ was $0.6 \mathrm{~mm}$ in the worst case where $l=50 \mathrm{~mm}$, but one can attain the tolerance about $\Delta h \leq 0.1 \mathrm{~mm}$ which would decrease the modulation depth about 0.001 units at $3000 \mathrm{~cm}^{-1}$ and with $D=25 \mathrm{~mm}$. If one flexure end was mounted $0.2 \mathrm{~mm}$ away from its correct position in $x$ direction, the angle between the neutral axes of the flexures would be $\theta=$ $4 \mathrm{mrad}$ with the porch swing $l=h=50 \mathrm{~mm}$, which was

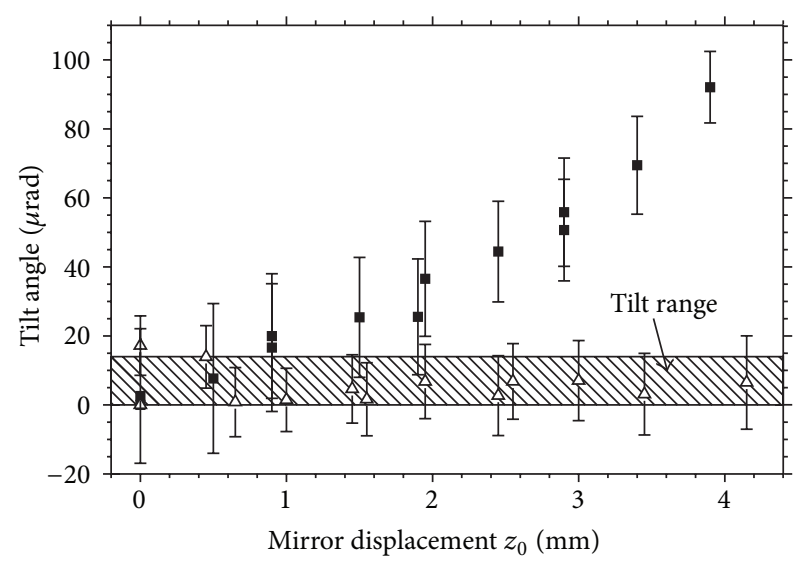

- No stiffening clamps

$\Delta$ With stiffening clamps

FIGURE 6: The stiffener clamps mounted to the spring strips decreased the tilt angle of the movable arm of the porch swing. The shaded area is the range of allowed tilt angle $\alpha \leq 14 \mu \mathrm{rad}$. The zero of the displacement was the equilibrium position of the spring elements. The error bars represent the maximum measurement error of the tilt angle. The maximum error of the mirror displacement was about $\pm 0.1 \mathrm{~mm}$.

the worst case. This positioning accuracy is well possible in practise. According to (A.5) and (1), this causes 0.015 decline in the modulation depth. However, the more common arm length in FTIR is about $150 \mathrm{~mm}$ which leads to a decline of about 0.002 . Exerting the driving force to the movable arm is sometimes more practical than to the ideal position. According to (A.7), the force position $a=h+6 \mathrm{~mm}$ produced $14 \mu \mathrm{rad}$ tilt angle which decreased the modulation depth to about 0.94 in the worst case when the arms were $50 \mathrm{~mm}$ long. In the other cases, the tilt was below $8 \mu \mathrm{rad}$ and the modulation at least 0.99 . Although, these tolerances were very loose, even the resulted worst case modulation depth values were small enough.

Hatheway [21] has estimated the tilt angles over $300 \mu \mathrm{rad}$ using the worst case tolerances. These are much greater than our estimations above. This was mainly because of significantly larger values for the tolerances and longer $5 \mathrm{~mm}$ stroke. Incidentally, over $80 \%$ of these angle values came from the arm length error and the nonparallelism of the arm ends.

Clearly, the most essential properties of the porch swing are the length difference of the arms and the nonparallelism of the arm ends. This has already been noted by many authors, for example, by Walker and Rex [6] and Strait [14] who have designed adjustment mechanism to minimize these errors. However, the previous calculations show that adjustment mechanisms are not necessarily needed which simplifies the construction and may reduce manufacturing costs.

2.4. Measured Modulation Depth with Porch Swing Bearings. We have studied a few porch swings by measuring the modulation depth as a function of the mirror position. In the following, we present two examples of them. In the first example, the porch swing had dimensions in millimeters 


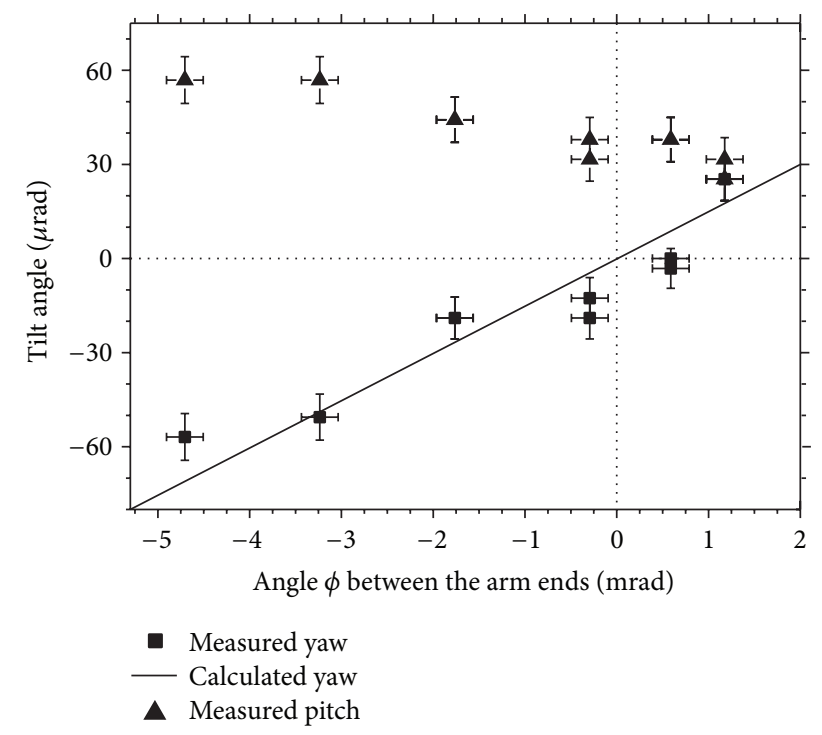

FIGURE 7: Measured pitch and yaw tilt angles and the calculated yaw angle from (A.6) as a function of the angle $\phi$ between the arm ends or the angle of nonparallelism of the inertia axes of the flexures. The error bars represent the maximum measurement errors estimated from the readings of the scales on the measurement equipment.

$l=133.4, h=50.0$, and $w=46.5$. Two flat springs were mounted in each end of the porch swing. We used the $30 \mathrm{~mm}$ stiffening clamps mounted in the halfway of flat springs. The porch swing was a part of the Michelson interferometer in a very similar setup as in Section 2.2, but the projection of the fringe pattern was not enlarged. The distances between the adjacent fringes were determined by using (2) and (3) and counting the number of fringes across the beam in vertical and horizontal directions at the mirror travel of $2 \mathrm{~mm}$ from the equilibrium position of the spring elements. After the first assembly, the porch swing caused the tilting a way too much to be used in an interferometer, thus some adjustment was required.

Firstly, we removed the yaw tilt by adding thin spacers under one flexure end which effectively lengthened the other side of the arm and thus changed the angle $\phi$ between the arm ends. The spacer thickness, which eliminated the yaw, was about $0.16 \mathrm{~mm}$. The flexures were then probably very close to parallel. The other measured spacer thicknesses and the corresponding values of $\phi$ were shifted so that the angle $\phi=0$ when the yaw tilt was about zero. The measured pitch and yaw tilt angles and the calculated yaw angles from (A.6) are presented in Figure 7. The measured yaw angles were in quite good agreement with (A.6). However, the pitch did not seem to be fully independent of the angle $\phi$, although the opposite could be expected. It is probably due to some uncertainty in the measurement. For example, only the upper arm ends were adjusted, so some errors might have remained in the lower arm dimensions.

Next, the pitch tilt was removed by adding more spacers under both springs on the same end of the upper arm, while keeping the difference of thickness between the two spacer stacks at about $0.16 \mathrm{~mm}$ to get nearly zero yaw. The pitch

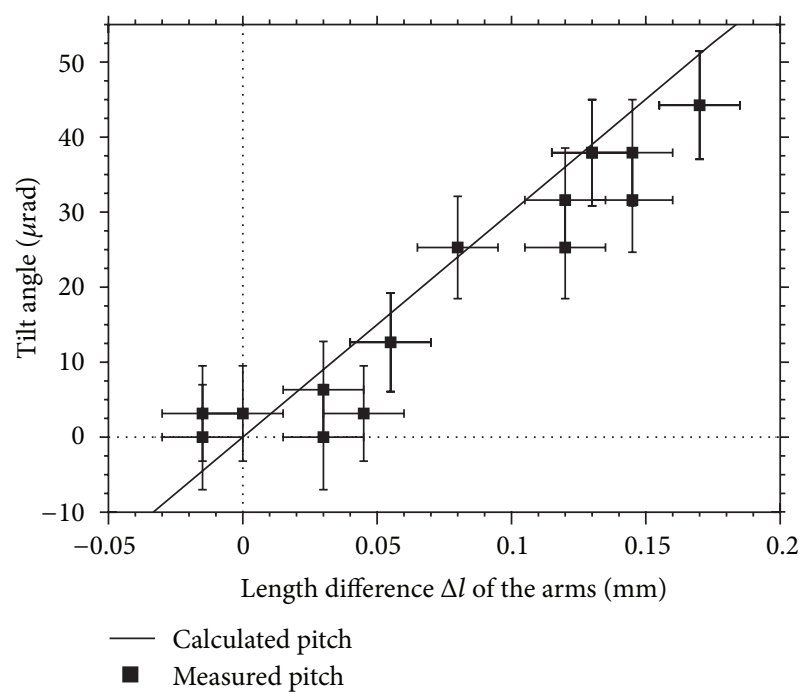

FIGURE 8: The measured and calculated values of the pitch tilt as a function of the length difference $\Delta l$ between the arms. The pitch was caused by the length difference of the arms. The error bars represent the maximum measurement errors estimated from the readings of the scales on the measurement equipment.

and the yaw were eliminated almost completely when the thicknesses of the spacer combinations were about $0.14 \mathrm{~mm}$ and $0.30 \mathrm{~mm}$. This corresponds to the length difference $\Delta l=$ $0.14 \mathrm{~mm}$. The pitch was also calculated using (A.1a) and (A.1b) which, among the measured values, is plotted in Figure 8. The measured length differences are shifted so that they are zero when the pitch is about zero. In addition to the tilt elimination, we obtained some support to the equations of the pitch. According to Figure 8, the measured pitch was increased with slightly smaller rate than the calculated pitch. However, there was still some yaw tilt almost every measurement point which among the other measurement uncertainties may have affected the results.

Slight tilting, which was left after the above fine tuning, was eliminated by replacing the $30 \mathrm{~mm}$ clamps with the $44 \mathrm{~mm}$ clamps and by careful reassembly and some minor changes of the spacers. Thus, the flexible parts shortened from $10 \mathrm{~mm}$ to $3 \mathrm{~mm}$. The changes in the fringe pattern were no more distinctive by the human eye, so the tilting was estimated by measuring the modulation depth of the interferometer output beam, which was focused on a photodiode as depicted in Figure 9. The movable arm was displaced by a pushing rod with fine threads. The zero position of the mirror displacement $z_{0}$ was the equilibrium position of the flat springs. In each mirror position, the rod mount was pushed carefully by hand to get a movement of a few fringes which caused a few complete sinusoidal cycles in the voltage signal from photodiode circuit. Because the photodiode was DC coupled, the positive minimum and maximum voltages $V_{\min }$ and $V_{\max }$ could be recorded. The visibility, or the modulation depth, is then about

$$
m \approx \frac{V_{\max }-V_{\min }}{V_{\max }+V_{\min }} .
$$




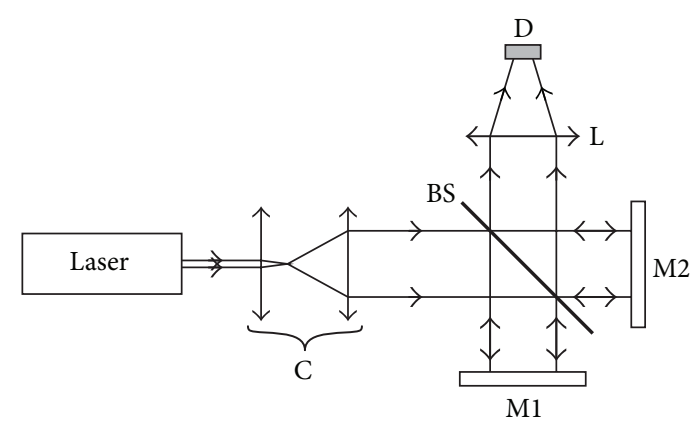

FIGURE 9: Measurement setup for determining the modulation depth of the output beam of the interferometer. The beam from Helium-Neon laser was collimated in $\mathrm{C}$ and then pointed through the Michelson interferometer built from plane mirrors M1 and M2 and a cube beam splitter BS. The mirror M2 was rigidly mounted and the mirror M1 was moved using the porch swing bearing. The converging lens $\mathrm{L}$ was used to focus the beam on the photodiode $\mathrm{D}$.

Although this is not the most accurate way to determine the modulation depth, hence, the noise is on these voltage values; our experience has shown that it gives a very good approximation especially when the signal has low noise as in this case.

The results of the previous modulation depth measurements are presented in Figure 10. In the first two measurements, the initial modulation depth was aligned to about 0.92 which is as close to 1 as possible with used optical components. Over $3.0 \mathrm{~mm}$ travel, the modulation depth was decreased not more than about 0.04 units. The result corresponds to about $4 \mu \mathrm{rad}$ tilt angle when the Gaussian distribution of laser intensity is considered [18]. This tilt is below the $14 \mu \mathrm{rad}$ limit set in Section 2.1. It would cause about 0.005 decrease in the modulation at $3000 \mathrm{~cm}^{-1}$ with a uniformly distributed beam which diameter is $25 \mathrm{~mm}$. In the other two measurements, an initial tilt was adjusted. The decrease of the modulation depth is roughly proportional to the squared tilt angle according to (1), so the initial tilt should cause a more rapid decrease in the modulation depth. However, this could not be observed which is a sign of very low tilting.

The above discussed porch swing was clearly machined poorly, because much adjustment was required. In the following example, the porch swing was assembled from properly cnc machined parts without modifying or tuning the parts in any way after the machining. The dimensions in millimeters were $l=110, h=53$, and $w=65$. The flat steel springs were $0.2 \mathrm{~mm}$ thick and $10 \mathrm{~mm}$ wide. The springs had the $51 \mathrm{~mm}$ stiffening clamps in the middle. The drawing scale of Figure 2 corresponds to these dimensions apart from the flexible parts which are exaggerated for clarity. The measurement setup was similar as in the previous experiment. The modulation depth of the interferometer was decreased about 0.05 units during the mirror travel of $3 \mathrm{~mm}$ as represented in Figure 11. This corresponds to about $5 \mu \mathrm{rad}$ tilt angle when the distribution of the laser beam is considered. The tilt is below the $14 \mu \mathrm{rad}$ limit set in Section 2.1. Using $25 \mathrm{~mm}$ beam, this tilt produces

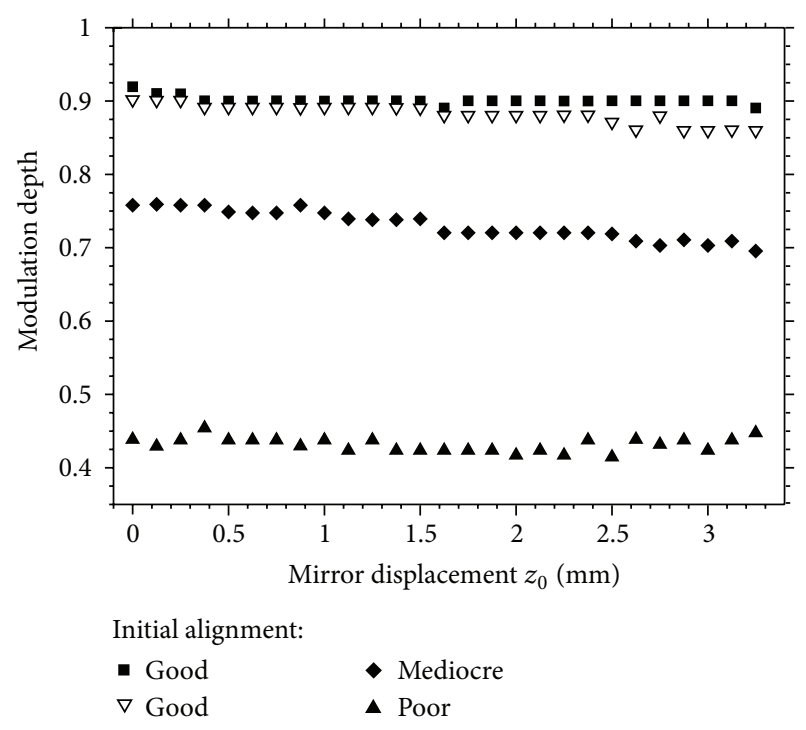

Figure 10: The modulation depth of an interferometer with welladjusted porch swing bearing. Four different initial tilt angles were used. The zero of the displacement was the equilibrium position of the spring elements. The maximum measurement error in the modulation is about 0.01 and in the position about $0.03 \mathrm{~mm}$.

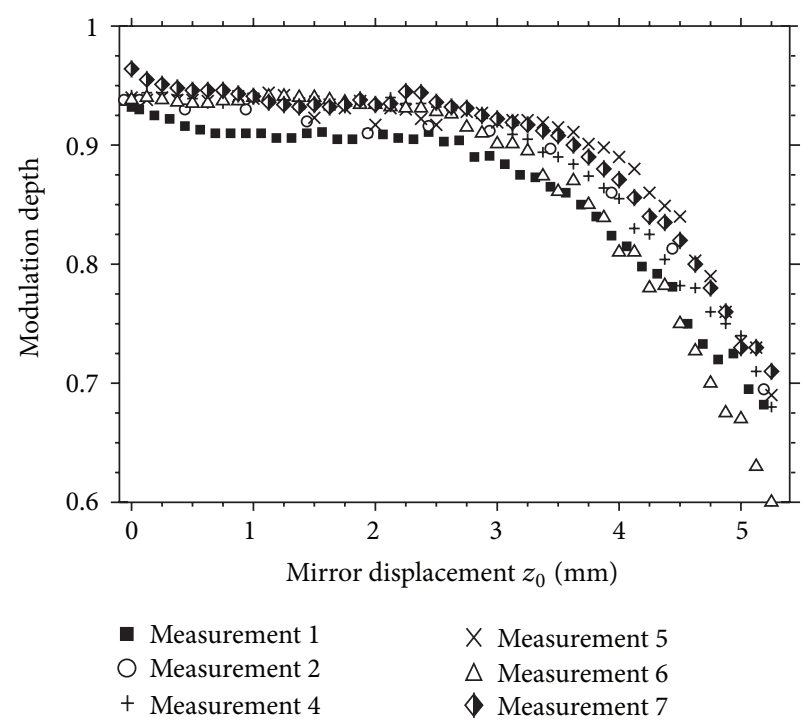

FIGURE 11: The modulation depth of the interferometer with a properly machined and carefully assembled porch swing. The zero of the displacement was the equilibrium position of the spring elements. The maximum measurement error in the modulation was about 0.01 and in position about $0.03 \mathrm{~mm}$.

about 0.007 decrease in the modulation depth at $3000 \mathrm{~cm}^{-1}$. The error in the determining of the modulation depth from the photodiode signal was about 0.01 . However, the results were not repeatable very well. This was probably because of the friction between the pushing rod and the upper arm although the friction was significantly reduced by a glass plate between the rod and the arm end as a slide bearing. 
Walker and Rex [6] used an interferometer which had the flexure pivot bearing as in Figure 1(c). They have reported that the tilting right after the assembly was about $150 \mu \mathrm{rad}$, but they achieved to decrease it to the acceptable value of $5 \mu \mathrm{rad}$ after adjusting of the pivot centers using the adjustment mechanism they had designed. Kemp and Huppi [10] used the similar interferometer and reported $5 \mu \mathrm{rad}$ maximum tilt over $5 \mathrm{~mm}$ travel but they did not mention if any adjustments were required. However, we think that they also might had to adjust the pivots to obtain sufficiently low tilting. As noted earlier, we have achieved the maximum tilt of $5 \mu \mathrm{rad}$ using only carefully machined parts without any tuning or adjustment mechanisms. The interferometer of Onillon et al. [26] used a porch swing, which maintained the tilt below $5 \mu \mathrm{rad}$ over $\pm 2 \mathrm{~mm}$ motion range and apparently had no arm length adjustment system, but it was not actually designed for FTIR spectrometer. Auguson and Young [27] have reported the tilting of five fringes after $1 \mathrm{~cm}$ travel. Their interferometer was, however, for far infrared and utilizing the ball bearings. With 3.75 inches beam they apparently used, the tilt was about $17 \mu \mathrm{rad}$, which would have been too much for mid-infrared.

Several authors have demonstrated the tilting of the porch swing bearings. Jones [7] has reported the tilt of $34 \mu \mathrm{rad}$ with a porch swing made with spring strips and asymmetrically mounted stiffening clamps. Hatheway [21] used monolithic flexures, where the flexure element and the clamps pressing it to the arm were machined in one piece. The smallest mentioned tilt values were pitch of $5 \mu \mathrm{rad}$ and yaw of $39 \mu \mathrm{rad}$. However, he has noted that these values may not always be repeatable because reassembling increased the tilt significantly. It seems that, both, Jones and Hatheway did not use the arm length adjustments. Muranaka et al. [20] have built a porch swing with adjustable arm lengths and have achieved the tilt angle less than about $0.5 \mu \mathrm{rad}$ with the maximum stroke of $\pm 3 \mathrm{~mm}$. However, their device is more appropriate for the demonstrations of the parasitic motions than as an actual bearing in an interferometer. Sizes of all above mentioned interferometers and demonstration bearings were comparable to example cases used in this article.

\section{Conclusion}

We have demonstrated experimentally that sufficient motion linearity of the mirror in Michelson interferometer is well achievable by using a porch swing bearing which has no adjustment mechanism which is often used. We defined the sufficient linearity by the maximum allowed decrease of the modulation depth which was 0.05 units over $3 \mathrm{~mm}$ mirror travel with $25 \mathrm{~mm}$ beam at $3000 \mathrm{~cm}^{-1}$. The corresponding decrease was achieved experimentally using Helium-Neon laser and a porch swing which was manufactured using normal machining and assembly tolerances. The estimated manufacturing tolerances for the porch swing were proven to be loose enough not to limit the application of the bearing in the FTIR interferometer. Additionally, the equations of the parasitic motions explained the tuning of the poorly machined porch swing.
We used the flat springs as the flexure elements of the bearing. We observed that the tilting was substantially decreased and the driving stability was improved by the stiffening clamps mounted in the middle of the springs. The clamps also increased the force constant or the spring rate of the bearing which might help in the vibration control of the system.

\section{Appendix}

\section{Equations of Parasitic Motions}

If the flexures are approximated as rigid links, as can be done with long stiffening clamps, and the arm lengths are $l$ and $l+\Delta l$ and the flexure lengths are $h$ and $h+\Delta h$, the structure looks like a quadrilateral as in Figure 3. The equations for the coordinates of the point $P_{2}$ can be derived by the intersection of two circles. The first circle is centered at $P_{0}$ and has the radius of $l+\Delta l$ and the second at $P_{1}$ with the radius of $h+\Delta h$. If the upper arm is shifted by $z_{0}$, the coordinates of the corner $P_{2}$ are

$$
\begin{gathered}
z_{2}=z_{0}+\frac{a}{d}\left(l-z_{0}\right)+\frac{k}{d} y_{0}, \\
y_{2}=\left(1-\frac{a}{d}\right) y_{0}+\frac{k}{d}\left(l-z_{0}\right),
\end{gathered}
$$

where

$$
\begin{gathered}
d=\left[\left(l-z_{0}\right)^{2}+y_{0}^{2}\right]^{1 / 2}, \\
a=(2 d)^{-1}\left[(l+\Delta l)^{2}-(h+\Delta h)^{2}+d^{2}\right], \\
k=\left[(l+\Delta l)^{2}-a^{2}\right]^{1 / 2} .
\end{gathered}
$$

Calculating the coordinates of $P_{2}$ at initial position $z_{0}=0$ and at displacement $z_{0}$ and assuming that the interferometer was perfectly aligned at its initial position, the pitch angle $\alpha$ can be calculated using basic geometry. Figure 12 illustrates pitch angles in several cases with typical porch swing dimensions and with the dimension error of $0.2 \mathrm{~mm}$ which can be regarded as an practical upper limit. The pitch seems to be proportional to $z_{0}$ with the $\Delta l$ but the dependence is approximately quadratic with $\Delta h$. It also seems that the error $0.2 \mathrm{~mm}$ in the arm length $l$ causes about 30 times bigger tilt angle compared with the equal error in the flexure length $h$.

Jones and Young [19] have presented approximations for the pitch angle as

$$
\begin{aligned}
& \alpha \approx-\frac{z_{0} \Delta l}{h l}, \quad \text { if } \Delta h=0, \\
& \alpha \approx+\frac{z_{0}^{2} \Delta h}{2 h^{2} l}, \quad \text { if } \Delta l=0 .
\end{aligned}
$$

The approximations are valid if the displacement $z_{0}$ is small. With relatively large displacements, the actual flexure lengths should be replaced with the effective lengths of $(5 / 6) h[20$, 

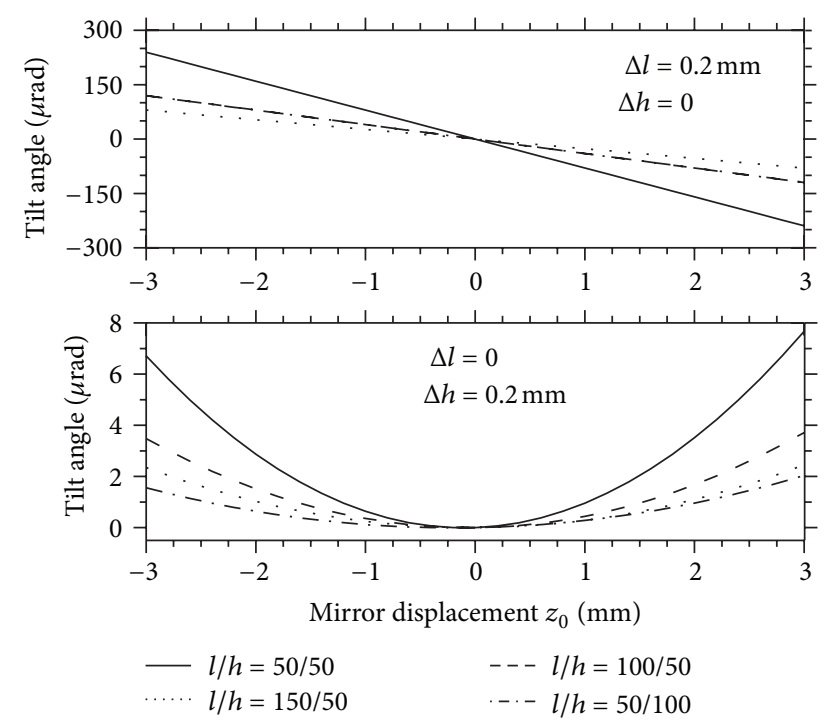

FIGURE 12: The pitch angle $\alpha$ as a function of mirror displacement $z_{0}$ calculated using (A.la) and (A.1b). In the upper graph, only the arm length error is considered, and in the lower one, only the flexure length has error.

22]. If we compare (A.3) and (A.4), we obtain the ratio of the length errors $\Delta h / \Delta l=2 h / z_{0}$. Using dimensions of a typical design, the ratio is about $(2 \times 50 \mathrm{~mm}) /(3 \mathrm{~mm}) \approx 30$, which agrees with the values in Figure 12 calculated from (A.1a) and (A.1b).

We compared these approximations numerically with our model using the same cases as in Figure 12 and noted that if there is only $\Delta l=0.2 \mathrm{~mm}$ or both $\Delta l=\Delta h=$ $0.2 \mathrm{~mm}$, the pitch from (A.3) differs from (A.1a) and (A.1b) less than about $1 \%$. However, (A.4) differed even tens of percent which was a result mainly from very small numerical values. The measurement results of Jones and Young [19] and Muranaka et al. [20] have fitted quite well to both the pitch approximations and our model.

The nonparallelism of the neutral axes of the flexure elements may happen, for example, if the flexures are rotated to each other by the angle $\theta$ around the $z$ axis. According to Hatheway [21], the yaw angle is

$$
\beta=\frac{\theta z_{0}^{2}}{2 l h}
$$

The effective length of the flexure has to be considered in this equation when necessary. The principal axes of inertia of the flexures become nonparallel when the flexure elements are rotated to each other by the angle $\phi$ around the $y$ axis which is the case if the ends of the arms are not parallel. Hatheway [21] has given the yaw angle as

$$
\beta=\frac{\phi z_{0}}{l} .
$$

Ideally, the driving force lies along a line which is parallel to the $z$ axis and runs through the center of the cross-section on the $x y$-plane of the porch swing $[20,21]$. The nonideal alignment of this force causes the parasitic rotations which have to be usually analyzed numerically [21]. However, some approximations are presented by Jones and Young [19] and Muranaka et al. [20], whose model is

$$
\alpha=z_{0} \frac{2(h-2 a) t^{2}}{l^{2} h^{2}}\left[1+\frac{3}{175}\left(\frac{z_{0}}{t}\right)^{2}\right],
$$

where $t$ is the thickness of the flat spring and $a$ is the distance of the force $\mathbf{F}$ from the lower arm as in Figure 1(a). It can be readily seen that the pitch angle is zero if $a=h / 2$. The tilting from the misaligned driving force is, however, usually much smaller than the other errors of the movement as noted by Jones and Young [19] and by us.

\section{References}

[1] R. R. Jackson, "Continuous scanning interferometers for midinfrared spectrometry, chapter: Instrumentation for Mid- and Far-infrared Spectroscopy," in Hand-Book of Vibrational Spectroscopy, J. M. Chalmers and P. R. Griffiths, Eds., vol. 1, pp. 264282, John Wiley \& Sons, 2002.

[2] J. Kauppinen, J. Heinonen, and I. Kauppinen, "Interferometers based on the rational motion," Applied Spectroscopy Reviews, vol. 39, no. 1, pp. 99-130, 2004.

[3] P. R. Griffiths and J. A. de Haseth, Fourier Transform Infrared Spectrometry, John Wiley \& Sons, 2nd edition, 2007.

[4] D. R. Nohavec, L. S. Schwartz, and D. L. Trumper, "SuperHybrid Magnetic Suspensions for Interferometric Scanners," JSME International Journal Series C, vol. 40, no. 4, pp. 570-583, 1997.

[5] H. Kobayashi, "Interferometric monitor for greenhouse gases (IMG)-project technical report," Tech. Rep., IMG Mission Operation \& Verification Committee CRIEPI, 1999.

[6] R. P. Walker and J. D. Rex, "Interferometer design and data handling in a high vibration environment-part I: interferometer design," in Multiplex and/or High-Throughput Spectroscopy, G. A. Vanasse, Ed., vol. 191 of Proceedings of SPIE, pp. 88-91, August 1979.

[7] R. V. Jones, "Parallel and rectilinear spring movements," Journal of Scientific Instruments, vol. 28, pp. 38-41, 1951.

[8] G. W. Small, R. T. Kroutil, J. T. Ditillo, and W. R. Loerop, "Detection of atmospheric pollutants by direct analysis of passive fourier transform infrared interferograms," Analytical Chemistry, vol. 60, no. 3, pp. 264-269, 1988.

[9] R. J. Huppi, R. B. Shipley, and E. R. Huppi, "Balloon-borne fourier spectrometer using a focal plane detector array," in Multiplex and/or High-Throughput Spectroscopy, G. A. Vanasse, Ed., vol. 191 of Proceedings of SPIE, pp. 26-32, August 1979.

[10] J. C. Kemp and R. J. Huppi, "Rocket-borne cryogenic Michelson interferometer," in Multiplex and/or High-Throughput Spectroscopy, G. A. Vanasse, Ed., vol. 191 of Proceedings of SPIE, pp. 135-142, August 1979.

[11] R. A. Hanel, B. Schlacman, F. D. Clark et al., "The Nimbus III Michelson interferometer," in Proceedings of the Aspen International Conference on Fourier Spectroscopy, G. A. Vanasse, A. S. Jn, and D. J. Baker, Eds., pp. 231-241, Air Force Cambridge Research Laboratories, Optical Physics Laboratory, United States Air Force, January 1971.

[12] E. H. Wishnow, R. Wurtz, S. Blais-Ouellette et al., "Visible imaging Fourier transform spectrometer: design and calibration," in Instrument Design and Performance for Optical/Infrared 
Ground-based Telescopes, M. Iye and A. F. M. Moorwood, Eds., vol. 4841 of Proceeding of SPIE, pp. 1067-1077, August 2002.

[13] G. L. Auth, "Ruggedized compact interferometer requiring minimum isolation from mechanical vibrations," US Patent 4,693,603, 1987.

[14] D. R. Strait, "Moving mirror tilt adjust mechanism in an interferometer," US Patent 4,991,961, February 1991.

[15] R. F. Lacey, "Support for a moving mirror in an interferometer," US Patent 4,710,001, December 1987.

[16] G. R. Walker, "Precision frictionless flexure based linear translation mechanism insensitive to thermal and vibrational environments," US Patent 6,836,968, 2005.

[17] K. C. Schreiber, "Support for a movable mirror in an interferometer," Patent Application Publication 2002/0149777, 2002.

[18] T. Välikylä and J. Kauppinen, "Modulation depth of Michelson interferometer with gaussian beam," Applied Optics, vol. 50, pp. 6671-6677, 2011.

[19] R. V. Jones and I. R. Young, "Some parasitic deflexions in parallel spring movements," Journal of Scientific Instruments, vol. 33, no. 1, article 305, pp. 11-15, 1956.

[20] Y. Muranaka, M. Inaba, T. Asano, and E. Furukawa, "Parasitic rotations in parallel spring movements," Bulletin of the Japan Society of Precision Engineering, vol. 25, no. 3, pp. 208-213, 1991.

[21] A. E. Hatheway, "Alignment of flexure stages for best rectilinear performance," in Optomechanical and Precision Instrument Design, A. E. Hatheway, Ed., vol. 2542 of Proceedings of SPIE, pp. 70-80, July 1995.

[22] A. E. Hatheway, "The kinetic center of the cantilever beam," in Optomechanical Design and Precision Instruments, A. E. Hatheway, Ed., vol. 3132 of Proceedings of SPIE, pp. 218-222, 1997.

[23] S. T. Smith, Flexures-Elements of Elastic Mechanisms, Gordon and Breach Science Publishers, 1st edition, 2000.

[24] S. Awtar, Synthesis and analysis of parallel kinematic XY flexure mechanisms [Ph.D. thesis], Massachusetts Institute of Technology, 2003.

[25] S. Awtar, A. H. Slocum, and E. Sevincer, "Characteristics of beam-based flexure modules," Journal of Mechanical Design, Transactions of the ASME, vol. 129, no. 6, pp. 625-639, 2007.

[26] E. Onillon, S. Henein, P. Theurillat, J. Krauss, and I. Kjelberg, "Interferometer scanning mirror mechanism," in Mechatronic Systems 2002: A Proceedings Volume From the 2nd Ifac Conference, M. Tomizuka, Ed., Ifac Proceedings Series, International Federation of Automatic Control, Elsevier, 2003.

[27] G. C. Auguson and N. O. Young, "A liquid-helium-cooled Michelson interferometer," in Aspen International Conference on Fourier Spectroscopy, G. A. Vanasse, A. S. Jn, and D. J. Baker, Eds., pp. 281-288, Air Force Cambridge Research Laboratories, Optical Physics Laboratory, United States Air Force, January 1971. 



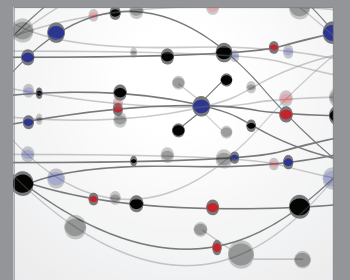

The Scientific World Journal
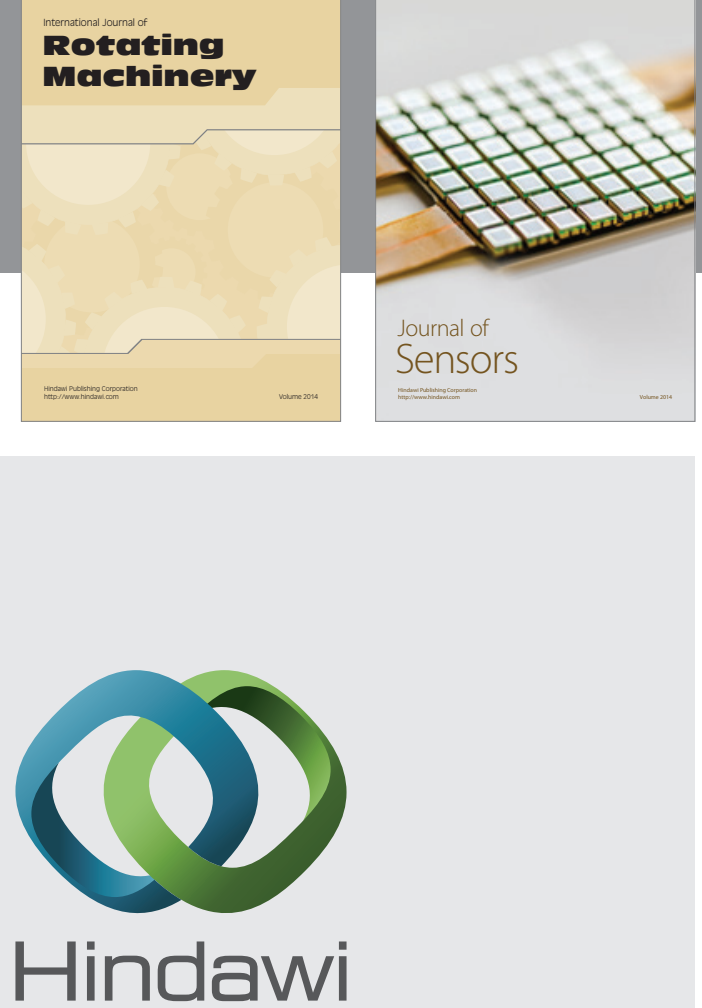

Submit your manuscripts at http://www.hindawi.com
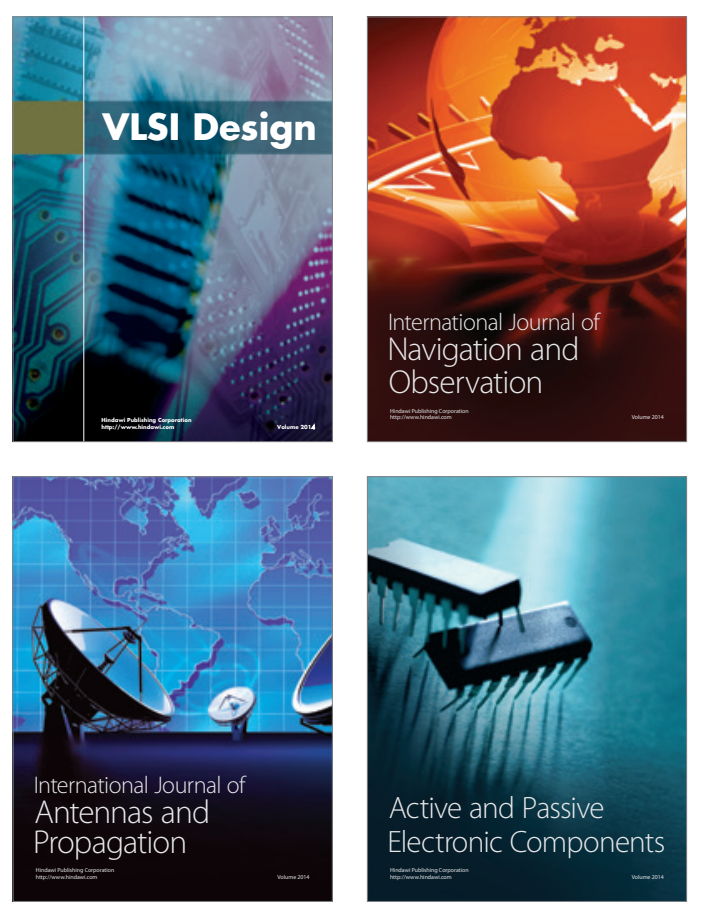
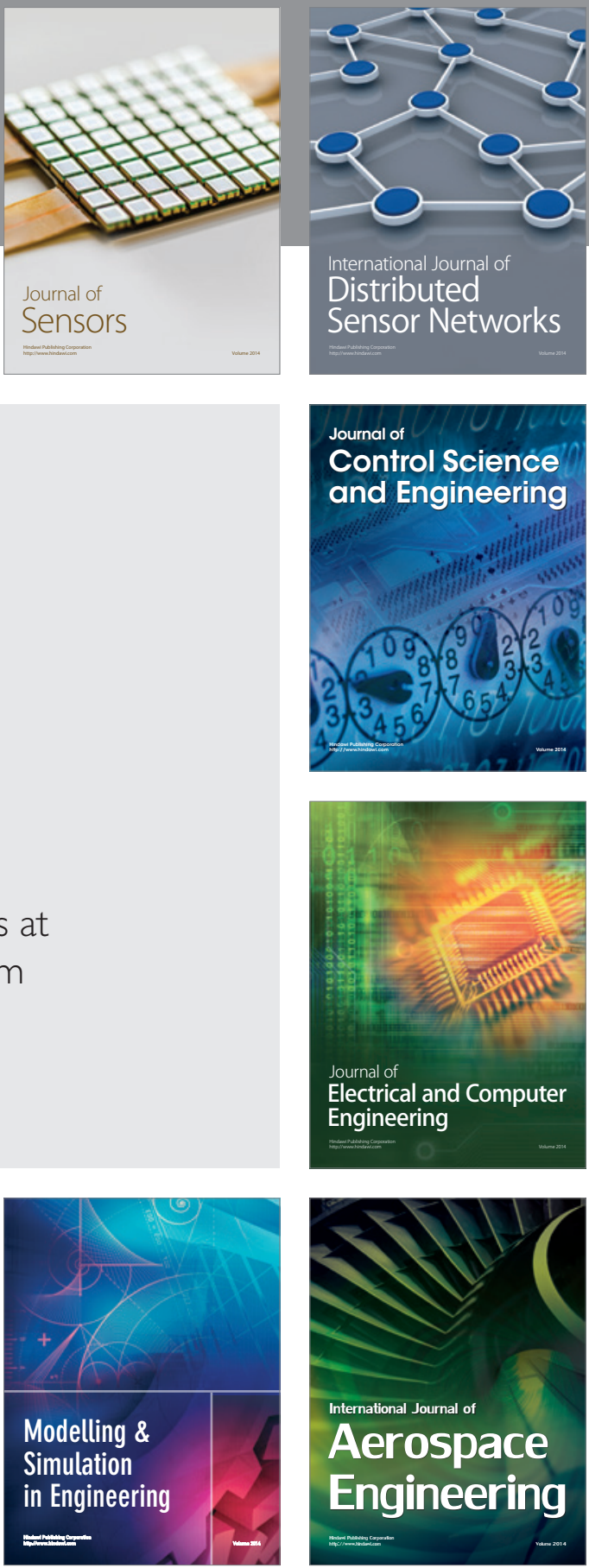

Journal of

Control Science

and Engineering
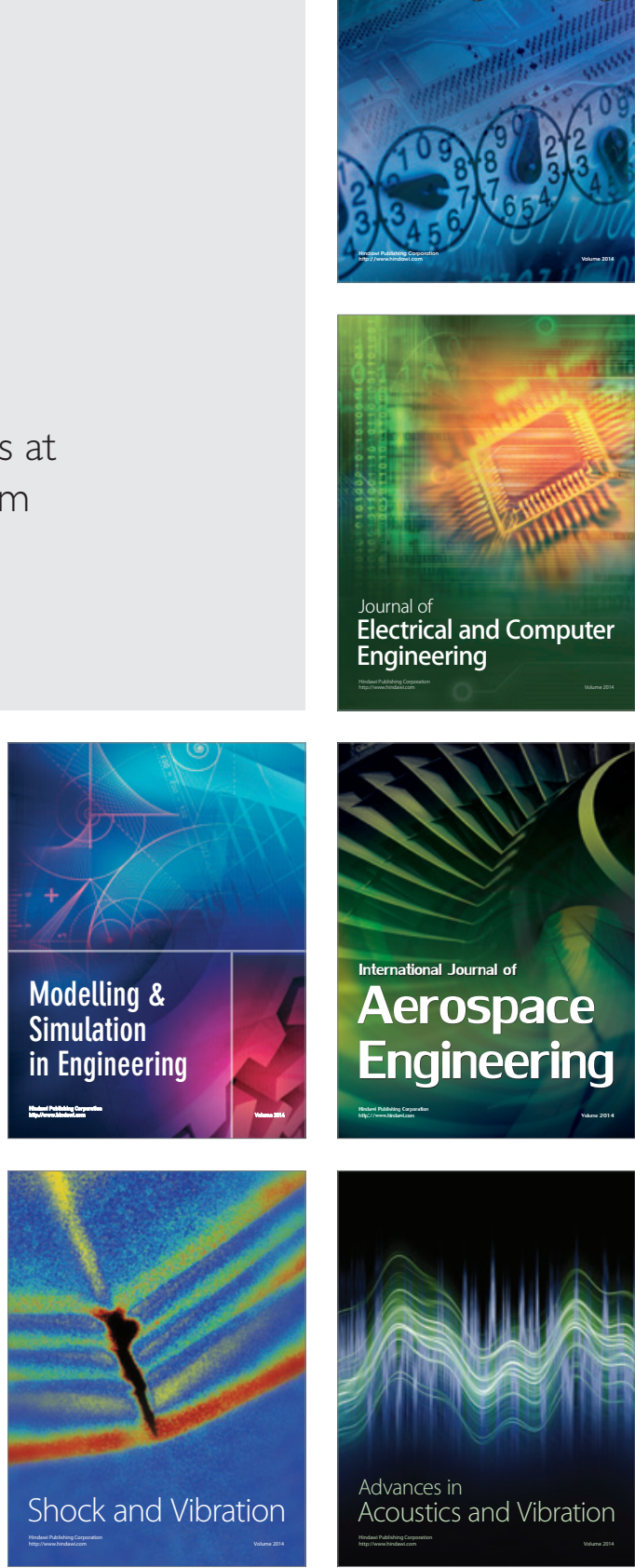